\title{
Empirically Derived Dynamical Models for the 55 Cancri and GJ 876 Planetary Systems
}

\author{
Benjamin E. Nelson ${ }^{1}$, Eric B. Ford ${ }^{1}$, Jason T. Wright ${ }^{1}$, and \\ Debra A. Fischer ${ }^{2}$ \\ ${ }^{1}$ Dept. of Astronomy \& Astrophysics, Pennsylvania State University \\ Center for Exoplanets and Habitable Worlds \\ 525 Davey Laboratory, University Park, Pennsylvania, USA, 16803 \\ email: benelson@psu.edu \\ ${ }^{2}$ Dept. of Astronomy, Yale University \\ New Haven, CT 06520 USA
}

\begin{abstract}
In this paper we present empirically derived dynamical models for the 55 Cancri and GJ 876 planetary systems.
\end{abstract}

Keywords. planets and satellites: individual (55 Cancri; GJ 876), radial velocity

55 Cancri and GJ 876 are two landmark radial velocity (RV) systems with 4+ planets. These systems display strong planet-planet interactions over their $\sim 10-20$ year observing baseline. Characterizing the orbits and masses of such planets can be difficult for a few reasons: covariance among model parameters, an oddly shaped posterior probability distribution, and the large dimensionality of the problem $(30+$ parameters $)$. Standard parameter estimation algorithms such as MCMC can be insufficient without some clever techniques. Even with a sophisticated algorithm, it becomes computationally tedious to perform an N-body integration for every model evaluation.

We apply our Radial velocity Using N-body Differential evolution Markov chain Monte Carlo code (RUN DMC; Nelson et al. (2014a)) to these two systems. The n-body integrations have been parallelized using SWARM-NG, a CUDA library to integrate an ensemble of few-body systems on GPUs. We perform subsequent long-term dynamical integrations using the MERCURY symplectic integrator (Chambers 1999).

55 Cancri: 55 Cancri is a Sun-like star hosting five known planets with a wide array of orbital separations and masses. We compile 1,418 unbinned RV measurements taken with the Lick Hamilton Spectrograph [Fischer et al. (2008)], HIRES [Fischer et al. (2008); Nelson et al. (2014b)], HET HRS, and HJST TS1 [Endl et al. (2012)]. In our orbital model, the outer four planets were assigned coplanar orbits and a separate inclination was given to the transiting inner-most planet $e$. Integrating the orbital evolution of 10,000 initial conditions drawn from our posterior samples for $10^{5}$ years, we find the mutual inclination between planet $e$ and the outer four planets must lie below $60^{\circ}$ or above $120^{\circ}$. The cause of these instabilities is planet $e$ undergoing Kozai-like perturbations, which pump its eccentricity such that the periastron of its orbit crossed the stellar surface. Accounting for relativistic precession in $e$ 's orbit causes the unstable domain in mutual inclination to widen slightly.

Planets $b$ and $c$ orbit near a 3:1 period commensurability. Over the timespan of the observations, we can detect the planets' orbits deviating from Keplerian behavior. We modeled the secular evolution of the 55 Cancri planets based on 10,000 sample systems. We find $36-45 \%$ of our model systems have $b$ and $c$ apsidally aligned about $180^{\circ}$ and undergo low amplitude libration $\left(51^{\circ} \pm_{10^{\circ}}^{6^{\circ}}\right)$. Other cases showed short-term perturbations 
in the libration of the secular angle, circulation, and nodding. We find the arguments associated with the 3:1 mean motion resonance are circulating over $99 \%$ of the time, so we conclude that these planets are not in resonance.

GJ 876: GJ 876 is an M dwarf hosting four known planets, where the outer three ( $c$, $b$, and $e$ ) are orbiting in a 1:2:4 resonant chain [Rivera et al. (2010)]. We compile 367 measurements from Keck HIRES [Rivera et al. (2010)], HARPS, and the ELODIE and CORALIE spectrographs [Correia et al. (2010)]. In our orbital model, we allowed every planet in the system to have its own inclination and longitude of ascending node.

The RVs meaningfully constrain the three-dimensional orbital architecture of all four planets. We see the mutual inclination between planets $c$ and $b\left(i_{c b}\right)$ has an upper limit of $\sim 7^{\circ}$ and $i_{b e}$ has an upper limit of $\sim 30^{\circ}$. We investigate the secular evolution of these models and find that higher mutual inclination in any planet pair typically leads to an instability. By demanding orbital stability, we infer that $i_{c b}$ has an upper limit of $\sim 4^{\circ}$ and $i_{b e}$ has an upper limit of $\sim 10^{\circ}$.

We can investigate the dynamical nature of this system by looking at the libration amplitude distribution of various angles associated with mean-motion resonances. Figure 1 shows these distributions for a coplanar (dashed) and full three-dimensional (solid) model. For at least one resonant argument associated with each planet pair, we find low or medium libration amplitudes, all of which are higher for the three-dimensional case than the coplanar case. Even so, these low amplitudes are strongly indicative of past disk migration of planets $c, b$, and $e$.

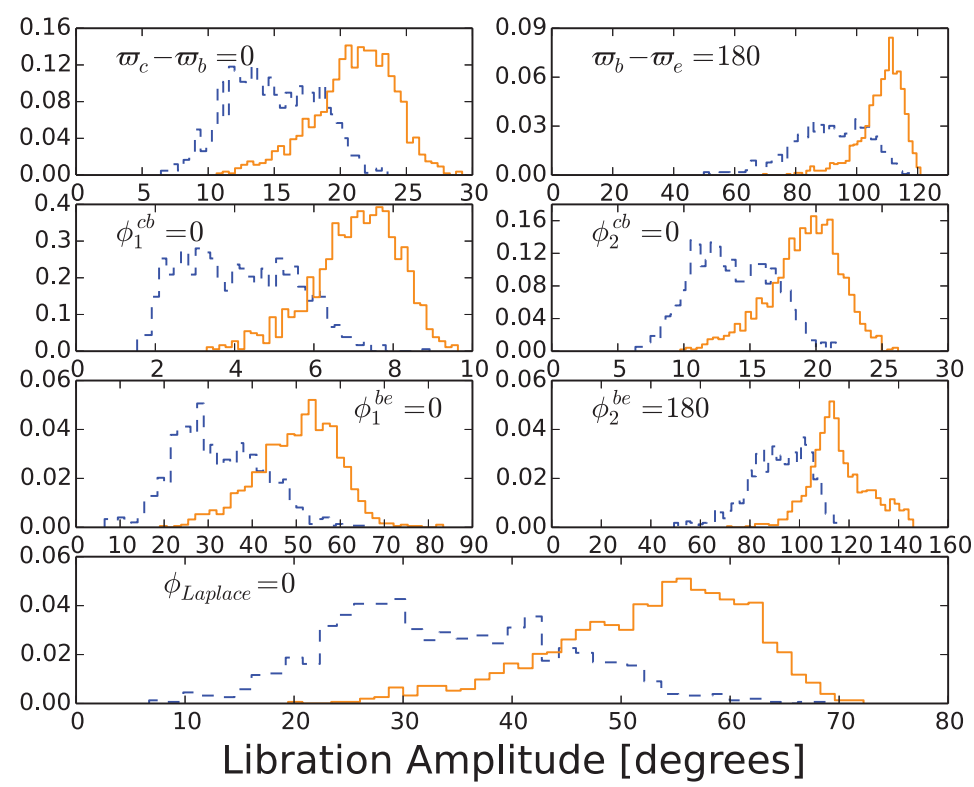

Figure 1. Libration amplitude distributions for various resonant arguments for a coplanar (dashed) and three-dimensional (solid) orbital model for GJ 876. Each panel shows an angle and what value it is librating about. First row, left: The apsidal alignment of $c$ and $b$ is librating about $0^{\circ}$. First row, right: The apsidal alignment of $b$ and $e$ is circulating. Second row: The two 2:1 MMR arguments for $c$ and $b$, both of which are librating about $0^{\circ}$. Third row: The two 2:1 MMR arguments for $b$ and $e$. The left panel is librating about $0^{\circ}$ and the the right panel is circulating. Bottom row: The Laplace argument is librating about $0^{\circ}$. 


\section{References}

Chambers, J. 1999, MNRAS, 304, 793

Correia, A. et al. 2010, A\&A, 511, 21

Endl, M. et al. 2012, ApJ, 759, 19

Fischer, D. et al. 2008, ApJ, 675, 790

Nelson, B., Ford, E., \& Payne, M. 2014, ApJS, 210, 11

Nelson, B. et al. 2014b, MNRAS, 441, 442

Rivera, E. et al. 2010, ApJ, 719, 890 